\title{
The Internal Structure of Semantic Relations: Effects of Relational Similarity and Typicality
}

Authors: Vencislav Popov', Margarita Pavlova² and Penka Hristova ${ }^{3}$

I Department of Psychology, Carnegie Mellon University, Pittsburgh, PA

2 Department of Psychology, University of Kansas, Lawrence, KS

3 Department of Cognitive Science and Psychology, New Bulgarian University, Sofia, Bulgaria

Correspondence: Vencislav Popov, vencislav.popov@gmail.com, 5000 Forbes Ave, Baker Hall, I5203, Pittsburgh, PA

Word count: 803I (excluding footnotes, bibliography and figures)

\begin{abstract}
We examined whether the processing of semantic relations shows typicality effects similar to those found for the processing of entity concepts. Participants performed four relational processing tasks with the same set of word-pair stimuli: relational exemplar generation; similarity ranking; analogical verification; and a paired-associate learning task. In the similarity ranking task, we gathered separate rankings for relational, role and semantic similarity between word pairs. We found significant correlations at the item level among relational generation frequencies, analogical verification RTs/accuracy and relational luring in associative memory. Relational similarity predicted exemplar generation frequencies, analogical verification RTs/accuracy, and relational luring in associative memory. Role similarity predicted exemplar generation frequency, and analogical verification RTs, but not relational luring. Semantic similarity did not predict any of the tasks, after controlling for the other two factors. Contrary to current theories which posit that semantic similarity is more important for retrieving relevant analogues, and that analogical mapping is based on role-filler bindings, relational similarity was the strongest predictor across all tasks. These results suggest that just like entity concepts, semantic relations have an internal structure that gives rise to typicality effects across a variety of tasks, which could provide constraints for testing competing theories of relational representation.
\end{abstract}

Keywords: semantic relations; analogical reasoning; similarity; memory 


\section{Introduction}

Human knowledge consists of both individual concepts (e.g. window or glass) and of semantic relations that connect these concepts with one another (e.g., the "is made of" relation between the concepts glass and window). Perhaps because they are easier to study, a focus on individual concepts has dominated the research on semantic memory and conceptual structures (Mahon \& Hickok, 2016; Murphy, 2002; Rosch \& Mervis, 1975; Rosch, Simpson, \& Miller, 1976), while questions concerning the nature and representation of semantic relations have been relatively ignored in comparison (Holyoak, 2007). This is unfortunate, because the ability to recognize and use such relational concepts has profound importance to the flexibility and productivity of human thought - relations provide the scaffolding for the conceptual system (Chaffin, 1992; Gentner, 2010), underlie the ability to abstract information beyond the entities that instantiate them (Gentner \& Kurtz, 2005; Hofstadter \& Sander, 2013), and may be considered as unique to human intelligence (Penn, Holyoak, \& Povinelli, 2008). While there have been theoretical debates concerning whether or not individual relations are represented explicitly, i.e. independently of the concepts they connect (Doumas \& Hummel, 2005; for a review, see Popov, Hristova, \& Anders, 2017), empirical research has only recently started to address these questions systematically (Asmuth \& Gentner, 2016; Chiang, Peng, Lu, Holyoak, \& Monti, 2019; Estes \& Jones, 2006; Gagné, Spalding, \& Ji, 2005; Popov et al., 2017).

The present study aims to explore how the typicality of the relational exemplars affect explicit relational processing in a range of tasks, such as analogical verification, associative recognition, relational exemplar generation and similarity ranking. Typicality/similarity norms derived from studies of relational reasoning have been used to predict relational

priming in short-term memory (Estes \& Jones, 2006), to predict relational luring in longterm memory (Popov et al., 2017), to predict patterns of neural responses to semantic relations in fMRI research (Chiang et al., 2019), and to train and test models of relational representations (Jurgens, Turney, Mohammad, \& Holyoak, 2012; Lu, Wu, \& Holyoak, 2019b). Thus, just like with entity categories, data for typicality and similarity of semantic relations has proven to be useful for model testing and development.

Nevertheless, research on relational similarity is much sparser in comparison to entity similarity, and it would be beneficial to gather more extensive data concerning how relational similarity affects processing in a variety of cognitive tasks, just like Rosch has done for entity similarity (Rosch \& Mervis, 1975; Rosch et al., 1976). The fact that entity typicality affects various processing tasks, including implicit ones, has supported the view that entity concepts have an internal structure that gives rise to such effects. In other words, typicality and similarity judgements are not simply the result of an artificial demand characteristic of the task - they reflect structural differences in the representations among concepts (Rosch, 1975; Rosch \& Mervis, 1975; Rosch et al., 1976). 


\section{Overview of the present study}

In order to study typicality effects for semantic relations, we selected four tasks that tested different aspects of relational processing for the same set of stimuli (see Figure 1): 1) a relational exemplar generation task, 2) an analogical verification task, 3) a continuous paired-associate learning task and 4) a similarity ranking task. We limit our investigation to the simplest form of relational processing - processing the similarity between single relations in a pair of items (e.g. verbal analogies of the type $A: B:: C: D$ or BIRD:NEST::BEAR::CAVE). Each task was completed by a different sample of participants, and those included both convenience samples of undergraduate students, as well as more heterogeneous samples recruited on social media.

\section{Experiment I}

\begin{tabular}{|cl|}
\hline Relational exemplar generation \\
\hline cues: & $\begin{array}{l}\text { airplane-pilot } \\
\text { horse-rider }\end{array}$ \\
\hline responses: & $\begin{array}{l}\text { car-driver } \\
\text { ship-captain } \\
\text { train-engineer }\end{array}$ \\
\hline
\end{tabular}

\begin{tabular}{|cc|c|}
\hline Associative recognition & Analogical verification \\
study: & test: \\
$\begin{array}{cc}\text { airplane-pilot } \\
\text { car-rug } \\
\text { floor-driver }\end{array}$ & $\begin{array}{c}\text { airplane-pilot } \\
\text { car-driver } \\
\text { floor-rug }\end{array}$ & airplane:pilot :: car:driver ? \\
\hline
\end{tabular}

\section{Experiment 2}

\section{Rank order similarity}

\begin{tabular}{cl} 
base: & airplane-pilot \\
\hline \multirow{2}{*}{ targets: } & $\begin{array}{l}\text { car-driver } \\
\text { ship-captain } \\
\text { train-engineer }\end{array}$
\end{tabular}

Conditions:

I) how relationally similar are the targets to the base?

2) how similar is the role of the first word in the targets and the base?

3) how similar is the role of the second word in the targets and the base?

4) how semantically similar are the first words in the targets and the base?

5) how semantically similar are the second words in the targets and the base?

Figure I. Examples of the three relational processing tasks compared in Experiment I, and the rank order similarity task in Experiment 2.

The rest of the paper is organized as follows. First, in Section 3 we will describe the first three tasks, and show the item-level correlation patterns among them to establish that there is systematic variability in the way people process exemplars of semantic relations across tasks. Next, in Section 4, we will consider three factors that could explain the shared covariability across these tasks - the semantic pairwise similarity of the individual concepts, the pairwise similarity of the roles that these concepts play within each relation, and the second-order similarity of the relations themselves. To motivate the choice of these 
three factors as explanatory constructs, we will consider two issues: 1) what drives memory retrieval of relational exemplars, semantic or relational similarity, and 2) are relations represented by role-filler bindings or by relational symbols? In Section 5, we will describe how we measured these three similarity factors, and we will show which of them can account for item-level performance in the main three tasks.

\section{Experiment I}

The questions motivating Experiment 1 were these: Do the frequencies with which people generate relational exemplars predict the speed and accuracy with which other people detect analogies involving these exemplars? Do relational generation frequency and the speed with which people detect analogies involving these exemplars predict the ease with which representations of the relation can be accessed from long-term memory (i.e., the relational luring effect, Section 3.2)?

\section{I. Relational exemplar generation frequencies}

In order to determine which relational exemplars are more accessible from semantic memory, we used a relational exemplar generation task (Jurgens et al., 2012). This task resembles the category-exemplar production task (Rosch et al., 1976) and requires a search through semantic memory for exemplars of the semantic relation. Participants saw two word pairs (cues) that were representative of a semantic relation (e.g., AIRPLANE-PILOT and HORSE-RIDER represent the $x$-is-driven-by-y relation), and they had to generate three additional word pairs that had the same relation. The dependent measure in this task is the proportion of people who generate each exemplar, with the assumption that more frequently generated pairs are more accessible from semantic memory and are more representative of the semantic relation exhibited by the cues (generation frequencies are often used as a proxy for typicality, Mervis, Catlin, \& Rosch, 1976). We used two different pairs as cues, because there are many ways in which two individual words could be related, and presenting two exemplars narrows down the pool of potentially relevant relations ${ }^{1}$ (Thibaut \&French, 2016). This task could potentially allow us to test competing theories concerning how relations are retrieved from semantic memory. A subset of the generated exemplars served as stimuli for the other tasks.

\footnotetext{
${ }^{1}$ For example, even though the target relation in airplane-pilot is " $\mathrm{x}$-is-driven-by-y", the concepts are also related spatially: "y-is-in-x". Presenting the second cue (horse-driver) clarifies that the spatial relation is irrelevant.
} 


\section{I.I. Participants}

Seventy-nine people (58 female) participated. Participants were native Bulgarian speakers that were recruited on social media and were asked in turn to share the study link with their contacts. Their age ranged from 18 to 67 years $(M=38, S D=12)$; Participants had heterogeneous education backgrounds - 80\% had a bachelor's degree or higher, 16\% had a high-school diploma and $4 \%$ had not graduated from high school. They were offered a chance to win a gift card for finishing the full study and for giving very typical exemplars of a relation.

\section{I.2. Materials, procedure and design}

We selected 2 exemplars for each of 58 relations, which we had already determined to be good exemplars of their relation in a previous pretesting study (for details, see Popov \& Hristova, 2015). Materials were administered through an online survey platform (http://esurv.org). For each relation the two exemplars were presented together and we asked participants to generate up to 3 novel exemplars for each relation. For example, participants where given the following two word-pairs:

NURSE HOSPITAL

WAITER RESTAURANT

They were told that the two word-pairs are analogical, because they share the same relation. Namely, a "NURSE' works in a "HOSPITAL", just as a "WAITER" works in a "RESTAURANT". They were told that this is not a creativity test and that they should attempt to write down the first analogical word pair that comes to mind. The whole procedure took between 30 and 120 minutes, and participants were told that if they are having trouble with some examples, it is better to move on to the next and that even partial responses will be of use. Relations were presented in random order for each participant, thus even if participants gave up before completing the task, responses were equally spread among all relations.

\section{I.3. Data cleaning and stimuli selection}

All responses were spell-checked and manually inspected before analysis, to remove differences between singular and plural forms, alternative spellings, etc. For each relation we counted the proportion of participants who gave each exemplar in each position (first, second or third response). Each of the 58 relations was given at least one answer from 47 to 67 participants. Each relation received between 117 and 186 separate responses. On average, the unique responses per relation that were given by more than one person were 19 \pm 10 . Responses given by a single person were often nonsensical (random letter strings), so we excluded them (as is often done with word norms, e.g. Nelson, McEvoy, \& Schreiber, 2004). The final dataset consisted of a total of 1085 relational exemplars split into 58 
different relations. The full dataset has been successfully used as a benchmark to test a model of relational representations ( $\mathrm{Lu}$, Liu, Ichien, Yuille, \& Holyoak, 2019a), but has not been made openly available previously. The full dataset is now available at https://github.com/venpopov/relational typicality.

A subset of this full dataset was used in all of the following tasks, because we wanted to use only stimuli with unique words for the relational luring task and many of the exemplars in the full dataset shared common words (for more detail, see Popov et al., 2017). For each of the 58 relation groups, we first identified the 8 most dominant responses. Going from the least to the most dominant response across relations, we manually and iteratively removed exemplars that shared one or two of their words with exemplars to other relations, until only responses with unique words remained. Only 35 of the initial 58 relations had more than 1 generated exemplar left, and for each we selected the 3 most dominant responses. Combined with the original two exemplars, this resulted in 35 relations with 5 exemplars each.

For each relational group we calculated the proportion of people that generated it in first place. This measure reflects how typical the exemplar is of the relation (more typical exemplars are generated more frequently) and it was used in all analyses presented later.

\subsection{Relational luring in associative recognition}

As we noted in the introduction, there have been theoretical debates whether relations are represented explicitly in memory (Doumas \& Hummel, 2005; Halford et al., 2010; Popov et al., 2017), as assumed by most analogical reasoning models (Doumas, Hummel, \& Sandhofer, 2008; Gentner, 1983; Hummel \& Holyoak, 1997; Kokinov \& Petrov, 2001; Lu, Chen, \& Holyoak, 2012), or whether they are implicitly represented as part of the entities they connect, as assumed by many semantic memory models (Collins \& Loftus, 1975; Collins \& Quillian, 1969; Gagné et al., 2005; Leech, Mareschal, \& Cooper, 2008; Smith, Shoben, \& Rips, 1974).

A recently discovered phenomenon in episodic associative recognition memory, the relational luring effect ( $R L E$ ), provides evidence that individual relations such as "works in" are represented independently from their arguments (DOCTOR-HOSPITAL; Popov et al., 2017). Participants were asked to study a list of word pairs in an associative recognition task and to respond on each trial whether a pair of words was seen previously in the experiment (i.e, "old pairs"), whether the words were seen, but in different pairs (i.e., "recombined pairs"), or whether they were new. The old and recombined pairs were selected from the 35 relational groups identified in the relational exemplar generation task described above. People studied and responded to five different exemplars of each relation. 
In order to succeed in this task, participants have to remember not only the individual words, but also to remember which words appeared together in a pair. The results showed that studying some exemplars of the relation (e.g., WAITER-RESTAURANT, TEACHER-SCHOOL, MECHANIC-WORKSHOP) made it more likely that people will subsequently wrongly identify novel exemplars (DOCTOR-HOSPITAL) as having been seen before. Even when participants correctly identified these exemplars as novel, they took longer to do so. We call this result "relational luring", because having seen some exemplars of a relation lures people to think that novel exemplars of the relation were also seen. Crucially, this relational luring effect increased linearly with the number of different exemplars that people had studied prior to being tested on the novel exemplar. Popov et al. (2017) proposed that when participants see relational exemplars such as TEACHER-SCHOOL or WAITER-RESTAURANT, they strengthen a common representation of the works-in relation. Later, when they see a novel exemplar of this relation (e.g., DOCTOR-HOSPITAL), the same relational representation is accessed, which causes a false sense of familiarity, leading to false alarms and slower correct rejections. When more exemplars of the relation are studied, its representation becomes stronger, and it induces an even stronger false sense of familiarity during the test of the novel exemplar. This causes participants to slow down to overcome the conflicting information and respond correctly.

What is important for the current investigation is that the slope of the reaction time to novel exemplars (i.e., the slope of the RLE) as a function of the number of studied exemplars varied across the 35 relations used by Popov et al (2017). Here, we were interested in whether the slope of the RLE would be systematically related to the overall relational similarity among the exemplars of the relation. The slope of the RLE is an implicit measure of how easy it is to access a relational representation from long-term memory - a larger RLE slope reflects the fact that the relational information was stronger and more difficult to overcome in order to judge a novel exemplar as unstudied. It is an important question whether the RLE slope would be systematically related to performance in the other relational processing tasks.

For the current study, we extracted the slope of the RLE for each exemplar of the 35 relations used in Popov et al. (2017).

\subsection{Analogical verification speed and accuracy}

In contrast to exemplar generation, the familiar $A: B:: C: D$ analogy format does not require the retrieval of relational exemplars from semantic memory, because the exemplars are given to the participant. What it does require is an additional process of analogical comparison that is absent in the exemplar generation task - people have to compute the second-order similarity between the relation present in the base and target pairs, and decide whether the relations are sufficiently similar to make a positive response. Response 
times for individual pairs in this task are influenced by the speed of the comparison process, and accuracy reflects how good the relational match between the base and the target is. If relational retrieval and analogical comparison are both influenced by systematic differences in similarity between exemplars, then we should see a correlation between generation frequencies and analogical verification accuracy/RTs.

\subsection{Participants}

Twenty-eight undergraduate students (7 males) at New Bulgarian University participated

for partial fulfillment for course credit. All were native Bulgarian speakers whose age ranged from 19 to 54 years $(M=32.4, S D=11)$. We excluded one participant due to low accuracy (59\%).

\subsubsection{Materials}

The final set of stimuli consisted of 444 unique word pairs. Half of the pairs were taken from the relations selected in the exemplar generation task. The other half of the pairs were fillers used on non-analogical trials, and they were not examples of any of the relations we previously used. For each relation, two of the pairs were always used as bases in the analogical judgement task, and the remaining four were always used as targets. For example, the relation "lives in" was represented by two bases CHEETAH-SAVANNAH and DOLPHIN-SEA and four targets MONKEY-JUNGLE, CAMEL-DESERT, GOAT-MOUNTAIN and MOUSEFIELD. An analogical trial consisted of one base and one target from the same relational group (e.g. CHEETAH : SAVANNAH :: MONKEY : JUNGLE). Non-analogical trials consisted of an existing base pair, and a filler target pair, in which the words were either unrelated, or not related in the same ways as the words in the base (e.g. DOLPHIN : SEA :: ZEBRA : STRIPES).

\subsubsection{Procedure}

We used a verbal analogy judgement task (Bunge, Wendelken, Badre, \& Wagner, 2005) in which analogical trials were intermixed with the same number of non-analogical trials. Each trial began with a fixation cross for $500 \mathrm{~ms}$. The fixation was followed by two words that appeared one above the other in the middle of the screen for $3.5 \mathrm{~s}$. Next, a question mark appeared for $500 \mathrm{~ms}$, indicating that the participants will be asked to provide an answer for the following word pair. Next, two words appeared one above the other until a response was given, followed by a blank screen for $500 \mathrm{~ms}$. For each second word pair, the participants had to indicate whether the words in it were semantically related in the same way as the words in the preceding pair.

\subsubsection{Design}

The design was within-subject with the type of the target pair (analogical or non-analogical to the base) as a single factor. Stimuli were counterbalanced with a latin-square across participants so that each participant rated two trials with different bases and targets from 
each relational group (for a total of 4 pairs from each relation), and each possible pairing of a base and target from each relational group was rated by a quarter of all participants. Participants completed 148 trials within 20 min.

\subsection{Results}

\subsection{Exemplar generation frequencies predict verbal analogy and relational luring}

The exemplar generation frequency correlated with the speed and accuracy of verbal analogy judgements - the greater proportion of people generate that exemplar when cued with other exemplars of the relation, the more accessible the relation is during verbal analogies, $r(210)=-0.49, p<.001$ for RTs and $r(210)=0.25, p<.001$ for accuracy (Figure 2b). Similarly, the RLE was stronger for pairs that were generated by more people, $r(210)=$ $0.31, \mathrm{p}<.001$ (Figure 2a). These findings suggest that it is easier to find an analogy between exemplars that are more typical of the relevant relation, and that more typical exemplars lead to stronger relational luring in long-term memory.

\subsubsection{Verbal analogy RTs predict relational luring strength}

Given that the results so far suggested that both generation frequencies and verbal analogy RTs tap the same accessibility aspect of the relational representation, we should expect that verbal analogy RTs to also predict the strength of the RLE. The RLE was stronger for pairs that were more accessible during the verbal analogy task (Figure 2c). That is, when target word pairs were identified faster as exemplars of a given relation in the verbal analogy task, they were also more difficult to identify it as lures in the associative recognition task

$\left(\Delta\right.$ AIC $\left.=-4, \chi^{2}(1)=6.34, \mathrm{p}=0.012\right)$. This result suggests that whether relational luring will occur, and how strong it will be, depends on the accessibility of the relational representation.

\subsubsection{Verbal analogy $R T$ s predict associative recognition}

Prior research has demonstrated that pairs of semantically related words are remembered more easily than pairs of non-related words (Epstein, Phillips, \& Johnson, 1975; Popov, Zhang, Koch, Calloway, \& Coutanche, 2019) and that this effect is larger if the association strength of the two words in a pair is larger (Silberman, Bentin, \& Miikkulainen, 2007). Although existing models threat association strength as the weight of the connection between words (Silberman et al., 2007), Popov et al. (2017) suggested that association strength reflects the strength of the underlying semantic relation. If that is the case, we should expect that the accessibility of the relation as measured by verbal analogy RTs should predict associative recognition performance for exemplars of the relation. We reanalyzed the associative recognition RTs by including the average verbal analogy RT estimates for each relation as predictors in our existing mixed-effect regression of associative recognition times (for more details, see Popov et al., 2017). Indeed, related word 
pairs were recognized more quickly, when the average analogical verification RTs for all exemplars of that relation were faster, $\Delta$ AIC $=-3, \chi^{2}(1)=4.83, p=.028$. For example, when it is easier to confirm that exemplars of the "X works in $\mathrm{Y}$ " relation are analogical to one another, it is also easier to remember that one has recently seen an exemplar of that relation (Figure 2d). This suggests that the availability of the relational representation affects episodic memory retrieval.
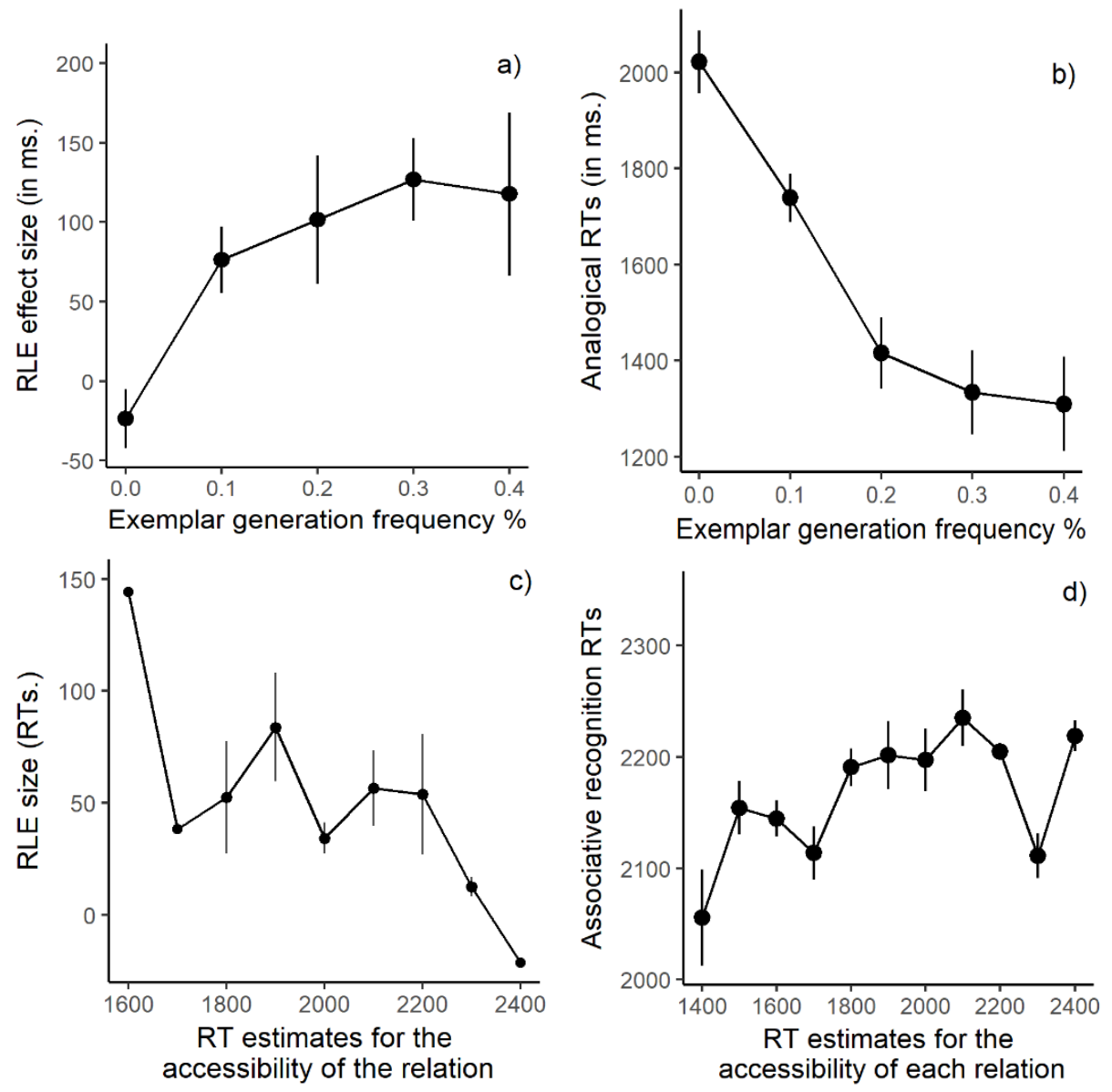

Figure 2. The effect of exemplar generation frequency on: a) the size of the RLE (i.e., the increase in RTs in ms. due to the number of previously studied exemplars of the relation; $b$ ) verbal analogy verification RTs. The effect of average analogical RTs for each relational group on the c) relational luring effect size and the d) overall associative recognition RTs

\section{Interim discussion}

Experiment 1 demonstrated that relational processing of the same word pair covaries across different tasks performed with that word pair. Specifically, the speed with which people can confirm that a word pair (HORSE-RIDER) shares the same relation as a base pair (AIRPLANE-PILOT), was faster if more people generate that pair as an exemplar of the relation $\mathrm{x}$-is-driven-by-y. These two measures (analogical verification speed and exemplar 
generation frequency) also predicted the speed with which people recognize previously studied exemplars in an episodic associative recognition task, and the degree to which they are lured by relationally similar exemplars in that task. It is important to note that these correlations were at the level of individual items, not individual participants, and that each task was performed by a different group of people. These results have an important implication - some properties of relational representations lead to covariability in relational processing, such that some relational exemplars are generated, compared and recognized better/faster than others. Thus, just like entity concepts, relational concepts have a graded internal structure that gives rise to typicality effects.

What representational properties can lead to the covariability we observed in experiment one? An obvious candidate is the degree of similarity either among relation exemplars, or between an exemplar and prototypical representation of a given relation (Chaffin \& Herrmann, 1984, 1989; Estes \& Jones, 2006; Ichien, Lu, \& Holyoak, 2019). Similarity is a central concept in cognitive science and it can be difficult to determine what stimulus features lead to differences in similarity (Gentner \& Markman, 1997; Goldstone, Medin, \& Gentner, 1991; Medin, Goldstone, \& Gentner, 1993; Sloman \& Rips, 1998; Tversky, 1977). Nevertheless, we can distinguish between three different kinds of similarity that could give rise to the effects observed in Experiment 1 - semantic, relational and role similarity.

\section{I. Semantic vs relational similarity}

Consider the following word pairs: AIRPLANE-PILOT, SHIP-CAPTAIN and HORSE-RIDER. Which one of them is most similar to the pair CAR-DRIVER? It depends on what features of the stimuli you take into account to make that decision. Gentner \& Markman (1997) distinguish between two types of similarity that influence comparisons - attribute (semantic) similarity and relational similarity. Semantic similarity reflects overlap between semantic attributes of individual concepts - a car could be considered more similar to an airplane than a horse, because both cars and airplanes are man-made, have wheels, have windows, etc. In contrast, relational similarity reflects overlap between relational attributes across pairs CAR-DRIVER could be considered more relationally similar to HORSE-RIDER than to either AIRPLANE-PILOT or SHIP-CAPTAIN, because they share relational attributes such as $x$-transports$y$-over-ground, $x$-usually-transports-small-numbers-of- $y$, etc (Chaffin, 1992).

There is mixed evidence regarding the degree to which semantic and relational similarity affect relational processing. Gentner (1983) showed that people judge two systems to be more similar if they share a common relational structure. Jones and Estes (2006) demonstrated that relational, but not semantic similarity predicts the strength of relational priming, i.e., the degree to which processing one relational instance (e.g., the word pair PLANET-CORE) influences the processing of a subsequent different instance of the same relation (e.g. FRUIT-PIT; Estes \& Jones, 2006; Popov \& Hristova, 2015; Spellman, Holyoak, \& 
Morrison, 2001). At the same time, there is evidence than when solving analogical problems, people often fail to spontaneously retrieve analogues from memory that share consistent relational similarities with the problem at hand, but rather depend on superficial similarity to retrieve information (Forbus, Gentner, \& Law, 1995; Gick \& Holyoak, 1980; Keane, 1987; Ross, 1989). Because of such findings, multiple analogical reasoning models have implemented the assumption that retrieving analogues from memory depends only on semantic similarity, and that relational similarity influences just the subsequent comparison processes (Forbus et al., 1995; Hummel \& Holyoak, 1997). In contrast, other models like AMBR (Kokinov \& Petrov, 2001), assume that retrieval can be driven by relational similarity as well.

It is important to note that this assumption, that relational similarity cannot contribute to retrieval (referred to as the relational retrieval gap by Holyoak, 2012), is based primarily on studies in which participants solved complex problems that consisted of multiple elements and relations among them. Following Dunbar (2001), Popov et al. (2017) argued that retrieval by relational similarity often fails in such tasks not because relational retrieval is impossible, but because representations of relations are often inconsistent across the base and target structures (for the same argument, see Jamrozik \& Gentner, 2020; Raynal, Clément, \& Sander, 2020). Nothing guarantees that when participants encode a complex multi-element base story they encode the relevant relations in the way they will do so for the target problem they have to solve.

If this explanation of the relational retrieval gap is correct, then relational similarity should predict analogue retrieval success when this issue of representational complexity and inconsistency is minimized. In the relational exemplar generation task, and in the relational luring associative recognition task, participants have to retrieve a word pair from memory, and this pair consist of only two elements, and the relations between them. These two tasks differ, however, in whether they require intentional or unintentional analogue retrieval. In the exemplar generation task, participants are explicitly instructed to generate exemplars of the same relation, thus relational retrieval there is intentional. In contrast, in the relational luring task, retrieval of the semantic relation is not only unnecessary - in fact, it impairs performance, because people falsely recognized novel pairs as having been seen before due to the shared relation with studied exemplars. Thus the relational luring effect represents an implicit measure of relational retrieval. It is an open question whether semantic or relational similarity would be a better predictor of retrieval in these two tasks.

\subsection{Relational vs role similarity}

In addition to semantic and relational similarity, conceptual structures can differ in the degree to which objects in them play similar roles within the structure (Jones \& Love, 2007). Roles can be considered to be structural components of relations - for example, the 
relation chasing involves two different roles - an agent (chaser) and a patient (chased). Even though we can verbally identify such roles within relations, it is not clear whether roles are actually included in the representations of relations and whether they are used during analogical processing. We will briefly discuss how current models of relational representation distinguish between relation-based and role-based representation.

Two major approaches have been proposed for representing relations explicitly and they are a matter of a prominent debate in the field (Doumas \& Hummel, 2005; Halford et al., 2010). In the relation-based approach, relations are usually represented by predicate-like entities, in which the arguments to the relation are specified by their spatial/order positions. For example, to represent the fact that a handle is part of a cup, one could posit three representational entities - $\mathrm{R}, \mathrm{x}$ and $\mathrm{y}$, where $\mathrm{R}$ stands for the part-of relation, and $\mathrm{x}$ and $y$ stand for the part and whole arguments respectively. Thus $x$ Ry or $R(x, y)$ represent the relational exemplar and one determines which object is the part and which the whole by their order in the representation. These types of relation-based symbol representations can be isomorphically implemented as predicates, part-of(handle, cup) (e.g., Gentner, 1983), localist nodes in a network with separate entities representing the relation and its arguments (e.g., Anderson, 1983), or conjunctions of distributed vectors, where each vector stands for either the relation or its arguments (e.g. tensor products, Halford, Wilson, \& Phillips, 1998). It is important to note that roles are not explicitly represented in such a representation, and are instead implicit in the order of arguments.

Even though in most of these relation-based models, relations are represented as high-level symbols, there are exceptions. According to relational element theory (Chaffin, 1992), relations are represented as a set of simpler elements such as connectedness, symmetry, inclusion, etc. that distinguish one relation from another. These simpler elements can be represented as a vector of feature weights for each element. This theory presumes that when people make relational comparisons, they compute the similarity between the element feature vector in the relational exemplars and the prototype of the relation stored in LTM.

Alternatively, in role-based models relations are represented as a set of relational roles to which entities are bound. For example, rather than explicitly representing the part-of relation as a separate symbol, the relational information can be represented as two roles, whole and part, to which each of the objects is bound (e.g., whole\&CUP + part\&HANDLE). In distributed models, this is achieved when two vectors representing each role of a diadic relation are bound either by multiplication or by addition or synchrony of firing/activation to vectors representing the entities that fill those roles (e.g., Hummel \& Holyoak, 1997). Some researchers have argued that such role-filler bindings are fundamental for relational processing and it is the similarity of the roles, rather than that of an relational symbol, that influences processing (Halford et al., 2010). 
A recent model of relational learning and reasoning, the Bayesian Analogy with Relational Transformations model (BART; Chiang et al., 2019; Lu et al., 2012, 2019b) implements both distributed role-based representations, as in Hummel and Holyoak's LISA model (1997), but also distributed representations of relational features, as in Chaffin's (1992) relational element theory. BART learns semantic relations from exemplars of those relations by associating a relational vector and two feature vectors representing the two words by learning a set of relational weights that connects them (Figure 3). These relational weights are distinctly separated to represent the role for the first and the second word. At the same time, the relational vector is represented as probability distribution over different possible relations (Figure 4). The latter representation is akin to Chaffin's (1992) theory, although in that theory the elements of the relational vector are not different relations, but different relational features.

Despite this variability in the way current models represent relational information, there is currently no empirical evidence that would allow us to distinguish whether representations of semantic relations are based on either relational-symbols, role-filler bindings or both. Our goal was to attempt a first step in providing evidence towards this question.

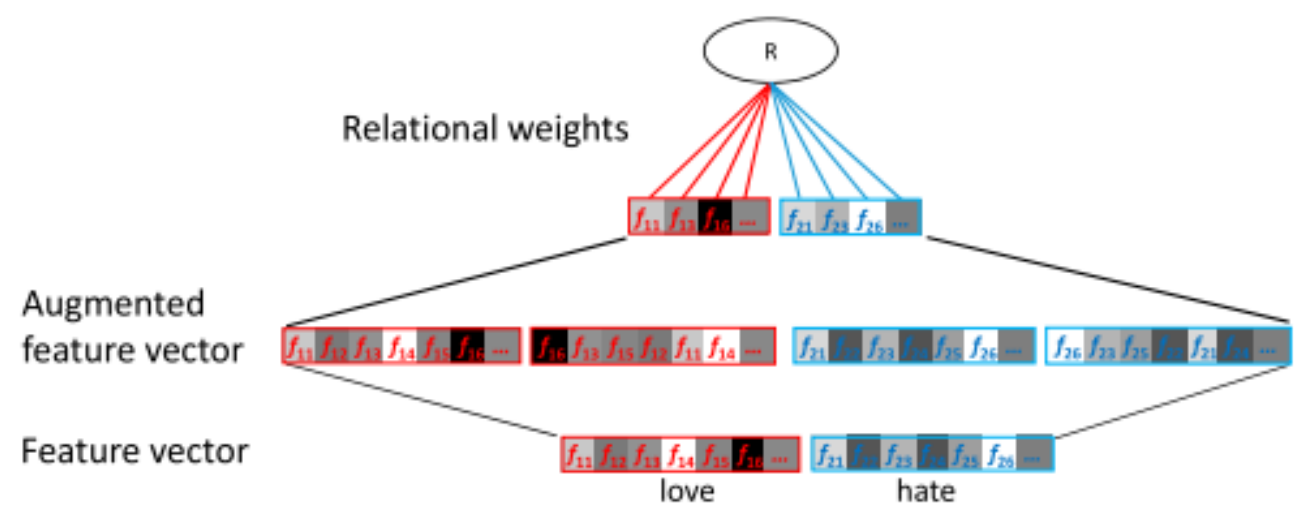

Figure 3. Illustration of the BART model for learning a semantic relation, $R$, from feature vectors for word pairs. Colors indicate features and weights associated, respectively, with the first word (red) and the second word (blue). Note that semantic roles (parts of networks based on first word and second word) are distinctly separated in the distribution of relation weights. Reproduced from Lu et al., 2019 with permission.

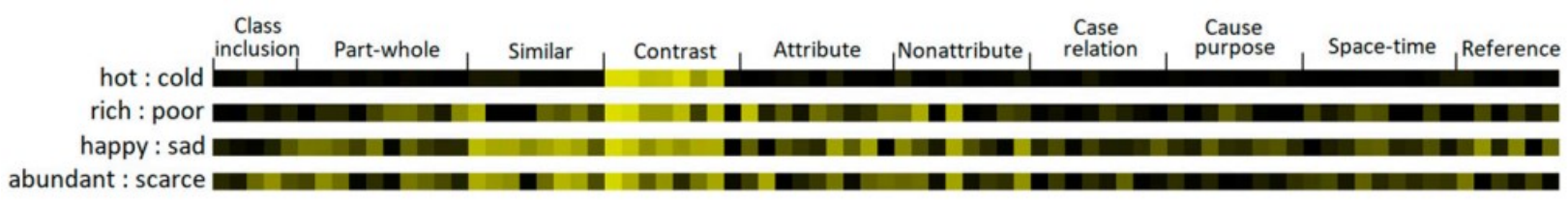

Figure 4. Illustration of relation vectors in BART. The relation present in each exemplar (different rows) is represented as a vector of probabilities that the exemplar instantiates each relation present in the model. Reproduced from Lu et al., 2019 with permission. 
Our exploratory approach was to present participants with pairs of exemplars from the same relation, and to ask them to separately rate the similarity of 1 ) the overall relation in the two exemplars or 2) the pairwise similarity of the roles each concept plays within the relation. It is not clear a priori whether participants would even be able to make these two judgements separately, or whether the result would be the same in both cases. A theory in which roles are not represented at all, would predict that while participants can rate the overall similarity of the relation between two exemplars, they would not be able to make separate judgements for the similarity of each role (e.g. how similar is the role that DOCTOR plays within the pair DOCTOR-HOSPITAL to the role WAITER plays in the pair WAITERRESTAURANT; and how similar is the role that HOSPITAL plays within the pair DOCTOR-HOSPITAL to the role restaurant plays in the pair WAITER-RESTAURANT). Instead, participants would base such judgements on the overall relational similarity between the pairs and or the simple semantic similarity between the objects. In contrast, a model like LISA, in which the representation includes separate features for each role, would predict that participants can make separate similarity judgements for each role; however, since the relation is represented entirely in terms of the two roles, the overall judgement of relational similarity would be entirely accounted for by the combined similarity of each of the two roles.

Thus, we explored two questions related to this issue: Can people make independent judgements of relational and role similarity? If they can, which of those two similarity types (in addition to semantic similarity), would be a better predictor of performance in the three tasks presented in Experiment 1?

\section{Experiment 2}

As a first step towards resolving the questions presented in Section 4, we asked participants to separately rank the semantic, role and relational similarity among different exemplars of the same relation. We gave participants one exemplar of a semantic relation (e.g. DOCTORHOSPITAL) as a base, and three other exemplars for them to rank order (WAITER-RESTAURANT, TEACHER-SCHOOL, WORKSHOP-MECHANIC) in one of five ways:

1) depending on how relationally similar they are to the base

2) depending on how similar the role of the first word in each exemplar is to the role of the first word in the base

3) depending on how similar the role of the second word in each exemplar is to the role of the second word in the base 
4) depending on how semantically similar the first word in each exemplar is to the first word in the base ${ }^{2}$

5) depending on how semantically similar the second word in each exemplar is to the second word in the base

\section{I. Method}

\section{I.I. Participants}

Fifty-two people (37 female) volunteered to participate. Participants were native Bulgarian speakers that were recruited through personal contact and social media. Their age ranged from 21 to 65 years $(M=35.0, S D=9.6) .82 \%$ held a bachelor's degree or higher.

\section{I.2. Materials, procedure and design}

The 35 groups of word pairs selected in the Relational exemplar generation task were split into five clusters of seven groups each. Each of the five clusters was assigned to be ranked by one of the following conditions using a latin-square design: 1) relational similarity, 2) role similarity of the first words in each pair, 3) role similarity of the second words in each pair, 4) semantic similarity of the first words in each pair, 5) semantic similarity of the second words in each pair

Each participant rated each cluster in only one of the five conditions and the conditions were randomized across participants such that each cluster was tested equally often in each condition. Half of the participants ranked the target pairs in response to the first base pair, and the other half ranked them in response to the second base pair. Thus, participants ranked 35 trials, which took them between 15 and 45 minutes. The task was administered through an online survey platform (Qualtrics.com).

On each trial, one of the base pairs in the group was presented on top (e.g. NURSE-HOSPITAL). Below it the three target pairs were shown (WAITER-RESTAURANT, LAWYER-LAWFIRM, MECHANICWORKSHOP) and participants had to rank how similar they are to the base according to one of the five criteria given above (semantic, role of relational similarity). Trials were presented in blocks of seven where all the trials in a block required the same criterion for ranking. The order of the blocks, the order of the trials within a block and the default order of the target pairs on each trial were randomized across participants.

${ }^{2}$ It could be argued that we would have obtained a purer measure of semantic similarity if participants rated individually the similarity of each pair of words, rather than seeing them in the context of other words. However, since the question is what contributes to processing precisely in the context of other related words, we decided to make the procedure the same for all ranking conditions, only changing the instructions. 
After participants completed the experiment, we asked them four questions during the debriefing:

a) Which of the three comparisons (semantic/relational/role) was most difficult to you? Rank them in decreasing order of difficulty

b) How confident are you about the rankings you gave for each criteria (on a scale from $1=$ completely unconfident to 5 = completely confident)?

c) Which two criteria did you have most difficulty distinguishing from one another? Rank them in decreasing order of difficulty (semantic from relational; relational from role; role from semantic).

d) On a scale from 1 (Completely indistinguishable) to 5 (Completely distinguishable), how difficult was it for you to distinguish the different criteria? Give a separate rating for each pair of criteria.

\section{I.3. Instructions}

Before each block, participants were instructed in detail as to how to rank the exemplars in each trial. The full instructions and examples are available in the Appendix.

\subsection{Results and discussion}

\subsection{Difficulty/discriminability of similarity rankings}

Participants gave separate rankings for the role and semantic similarities of each word within a pair, so we averaged the ranks over the two words into composite scores for overall role and semantic similarity rankings of each base-target pair. The rankings were centered and reversed to created similarity scores that ranged from -1 (least similar) to 1 (most similar).

We first looked at responses to our debriefing questionnaire in order to determine how difficult it was for participants to discriminate among the different types of similarity. Participants found it more difficult to discriminate the role from the other two criteria $(\mathrm{M}=$ $1.69, \mathrm{SD}=0.76$, against the relational criterion, $\mathrm{M}=1.82, \mathrm{SD}=0.8$, against the semantic criterion, $p=.47)$, compared to discriminating the relational from the semantic $(\mathrm{M}=2.49$, $\mathrm{SD}=0.64$, both $p$ 's <.001). Nevertheless, participants found the three ranking criteria to be equally difficult - Wilcoxon signed rank tests revealed that there were no significant differences in how difficult participants ranked the relational $(\mathrm{M}=2.16, \mathrm{SD}=0.79)$, role $(\mathrm{M}$ $=1.94, \mathrm{SD}=0.82)$ and semantic criteria $(\mathrm{M}=1.90, \mathrm{SD}=0.81)$, all $p s>.168$. Confidence scores for the three criteria were also similar $(\mathrm{M}=3.37, \mathrm{SD}=1.06 ; \mathrm{M}=3.39, \mathrm{SD}=0.85 ; \mathrm{M}$ $=3.39, \mathrm{SD}=0.80$; respectively for the relational, role and semantic criteria). 
Table I. Correlations among average rankings for each base-target pair for all criteria in Task 2.

\begin{tabular}{llllllll}
\hline & rel & role & sem & rolel & role2 & seml & sem2 \\
\hline rel & - & - & - & - & - & - & - \\
role & 0.62 & - & - & - & - & - & - \\
sem & 0.6 & 0.65 & - & - & - & - & - \\
rolel & 0.57 & 0.85 & 0.6 & - & - & - & - \\
role2 & 0.55 & 0.83 & 0.6 & 0.68 & - & - & - \\
sem 1 & 0.59 & 0.64 & 0.77 & 0.62 & 0.56 & - & - \\
sem2 & 0.6 & 0.62 & 0.77 & 0.53 & 0.62 & 0.53 & - \\
\hline
\end{tabular}

* Rel = relational rankings; Role and Sem are composite rankings, averaged over the two words in each pair; rolel, role2, seml and sem2 are the rankings based on the first and the second word in each pair, respectively

The three types of similarity for each base-target pair correlated moderately with each other, which suggests that participants were able to distinguish among them. Table 1 shows the Kendal tau rank correlation, calculated separately for each base-target group and averaged over groups. In summary, all rankings were equally difficult and participants were able to evaluate separately the semantic, role and relational similarities between word pairs, although their ratings were correlated with each other. There are two possible reasons for the inter-correlations among the different similarity ratings. First, it is possible that our stimulus set contains word pairs for which these three metrics are correlated in their representations. Thus, the inter-correlation among rankings could be due to the natural correlation present in the stimuli representations. Second, it is possible that even if semantic, role and relational similarity are orthogonal in the stimuli, that people were somewhat influenced by all three similarity sources in making their rankings. We cannot distinguish between these two possibilities.

\subsubsection{Relational and role similarity independently predict generation frequencies}

All similarity measures correlated positively with exemplar generation frequency. The correlation for relational similarity was $r(195)=0.44, \mathrm{p}<.001$; for role similar it was $r(195)=0.41, \mathrm{p}<.001$; and for semantic similarity it was $r(195)=0.35, \mathrm{p}<.001$.

Since the three similarity measures correlated with each other as well, we compared all three as predictors in a mixed-effects logistic regression model of the generation frequency proportions. We used a mixed effects regression model, rather than just partial correlation, because the observations for each pair of word pairs are not independent, but are nested within each relational group. The mixed effects regression had random intercepts for each relational group and random slopes for the three similarity measures within each relational group as well. After controlling for the other two factors, relational similarity significantly 
predicted generation frequencies, $\Delta$ AIC $=-15, \chi^{2}(1)=16.87, p<.001$. The effect of role similarity was also significant, but to a lesser degree, $\Delta$ AIC $=-2, \chi^{2}(1)=4.06, p=.044$. Semantic similarity had no effect, after controlling for the other two factors, $\Delta$ AIC $=-0.9$, $\chi^{2}(1)=2.84, p=.092$. The results for each measure after controlling for the other two are shown in Figure 5. In summary, when participants generated novel relational exemplars in response to a base pair, their memory search was guided by both relational and role similarity, but not by semantic similarity.
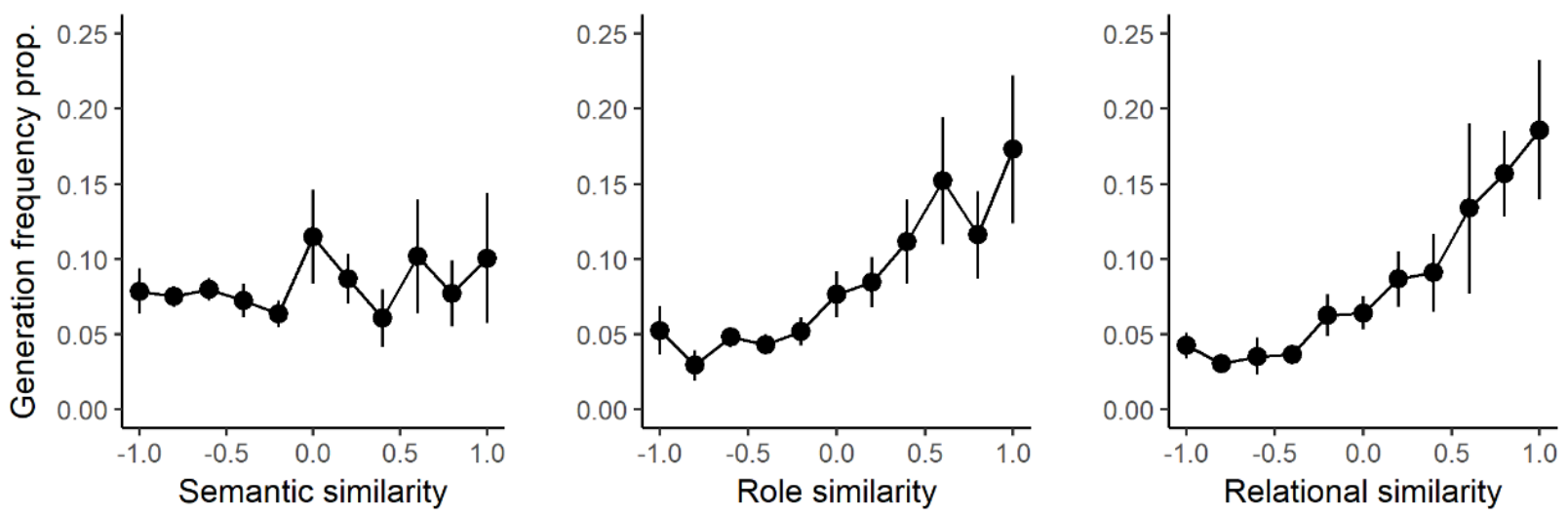

Figure 5. The effects of semantic, role and relational similarity on the frequency of generating relational exemplars. The results are shown after accounting for the effects of the other two factors. Each point represents the average generation frequency across pairs that received the same similarity score.

\subsubsection{Relational and role similarity independently predict analogical verification $R T s$}

The results were similar for analogical verification RTs and accuracy - all three similarity measures correlated significantly with analogical performance. For analogical RTs, the correlation was strongest for relational similarity, $r(210)=-0.38, p<.001$, followed by role similarity, $r(210)=-0.35, p<.001$, and weakest for semantic similarity, $r(210)=-0.31, p<$ .001). To contrast the three different measures, we used all three as predictors in a linear mixed-effects regression of analogical RTs, which included random intercepts for each relational group and for each subject, and random slopes for each of the three similarity measures within each relational group and within each subject. Relational similarity was the only significant predictor of analogical RTs, $\Delta$ AIC $=-2, \chi^{2}(1)=4.69, p=.03$. Neither role similarity, nor semantic similarity significantly predicted RTs after controlling for the other two measures $\left(\triangle \mathrm{AIC}=-1, \chi^{2}(1)=3.006, p=.083\right.$ and $\Delta \mathrm{AIC}=1.9, \chi^{2}(1)=0.023, p=$ 0.879 , respectively). Since semantic similarity was not a significant predictor, and the significance for role similarity was close to the $\mathrm{p}=0.05$ threshold, we rerun the model without the semantic similarity predictor. In this model, both relational and role similarity predicted analogical RTs (both p's < .05). The results for each measure after controlling for the other two are shown in Figure 6. In summary, greater relational and role similarity both 
lead to faster analogical decisions, although the evidence is stronger for the relational similarity contribution.
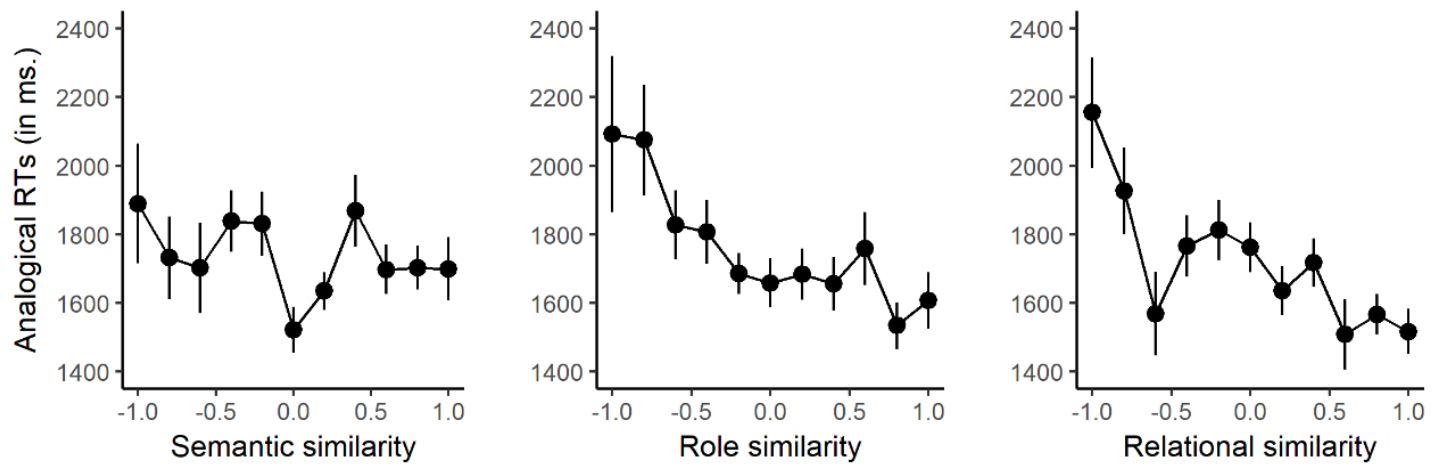

Figure 6. The effects of semantic, role and relational similarity on response times for analogical decisions. The results are shown after accounting for the effects of the other two factors. Each point represents the average analogical RTs across pairs that received the same similarity score.

\subsubsection{Only relational similarity predicts relational luring}

Relational luring represents the degree to which participants are influenced to falsely recognize a novel stimulus as previously seen because it implements the same relation as some of the studied stimuli. Only relational similarity correlated significantly with the strength of relational luring, $r(142)=0.20, p=.02$; the correlation with role and semantic similarity was not significant, both $r(142)=0.11, p=.18$. Once again, we also fit a linear mixed-effects regression model with random intercepts and slopes for each relational group, in which we could estimate the unique contribution of each similarity type. Confirming the correlation analyses, only relational similarity predicted relational luring, $\Delta$ AIC $=3.2, \chi^{2}(1)=5.35, p=.021$. The results are shown on Figure 7 . In contrast, luring occurs regardless of whether the words in the pairs are semantically dissimilar or whether they play dissimilar roles.
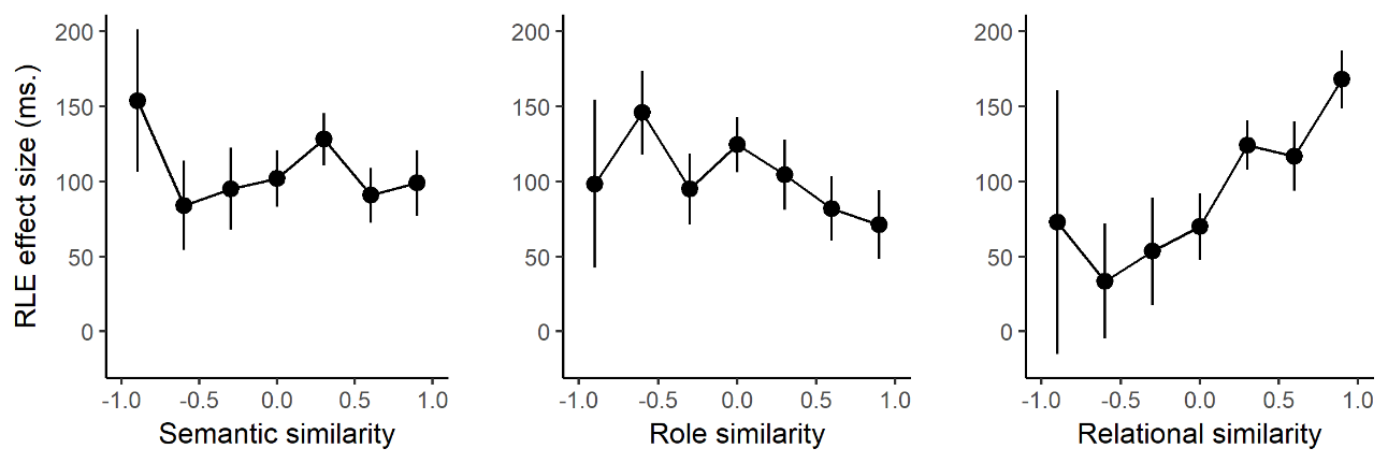

Figure 7. The effects of semantic, role and relational similarity on the strength of the relational luring effect (RLE). The results are shown after accounting for the effects of the other two factors. Each point represents the average relational luring across pairs that received the same similarity score. 


\section{General discussion}

We found that semantic relations exhibit typicality effects similar to those shown for entity concepts (Rosch et al, 1976). Experiment 1 showed that the frequency of generating a novel word pair exemplar of a semantic relation, the subjective relational similarity between exemplars, the speed of making analogical judgements, associative memory for relational exemplars and relational luring are all correlated with one another. These interdependencies were found at the item level and between answers of different groups of participants. This suggests there is a common representational factor that determines variance in relational processing. We argue that these effects of relational exemplar typicality provide evidence for a graded internal structure of relational concepts. Semantic relations are not only represented explicitly and independently from their constituents, as argued by Popov et al. (2017), but their accessibility is a function of their typicality: more typical instances have a priority access to the relational concepts compared to less typical instances.

These findings open a whole range of possible questions concerning the acquisition, the representation and the use of the relational concepts. If relational concepts stand for some shared invariance among its exemplars (Doumas et al., 2008) what is the essence of that overlap? Our results indicate that relational concepts need to be represented in such a way so as to be differentially accessed depending on the exemplar typicality. The exemplar typicality/similarity of entity concepts is usually accounted for with a greater featural overlap with the other exemplars from the same sematic category (Rosch \& Mervis, 1975). In contrast, the substrate of relational categories is argued to be captured by representations in terms of disembodied roles (Doumas et al, 2008), relational predicates (Anderson, 1983; Gentner, 1983; Halford et al, 1998) or a combination of a probability vector over possible relations that are connected in turn to a set of attribute feature vectors via relational weights separated for each role within the relation ( $\mathrm{Lu}$ et al, 2019b).

Findings from Experiment 2 are at odds with the pure role-based account that does not include explicit relational information. Across all tasks, relational similarity accounted for a greater amount of the variance in relational processing than role similarity. At the extreme, there was no evidence that role-based information contributes to implicit relational luring from long-term memory. Hence, the relational invariance argued to be at the core of the relational representations might not be bound to the role similarity as strongly as was previously suggested. These findings are also inconsistent with accounts that suggest role information is implicit in the order of arguments within a relational predicate (Anderson, 1983; Gentner, 1983). The fact that participants were not only able to independently evaluated the role and the relational similarity of exemplars, but also that these metrics independently predicted relational processing, suggests that both relational and role 
information must be present in the underlying representation, as assumed by models like BART (Lu et al, 2019).

When it comes to retrieval, we found no evidence for the claim that semantic similarity is a stronger driver of relational retrieval than relational similarity. Both explicit (exemplar generation) and implicit (relational luring) relational retrieval was better predicted by relational rather than semantic similarity, and only the former was significant after controlling for the latter, but not vice versa. This result sheds doubt on the existence of a relational retrieval gap, and suggests that failures in relational retrieval might be due to differences in the initial encoding of relations across exemplars (i.e., it is more an encoding gap; for a detailed argument, see Popov et al, 2017). In addition, since relational retrieval in the current study was superior for more typical exemplars, the usual retrieval gap may be a side effect of previous studies that focus on problem-solving, which by definition require finding a way out of a difficult, non-typical situation. Hence, even though a proper relation is encoded, the exemplar that instantiate it may be low in typicality and therefore incapable to access the target relation. That is why factors other than relational similarity, such as the base-target semantic similarity (Forbus et al., 1995; Gick \& Holyoak, 1980; Keane, 1987; Ross, 1989), may have explained performance better in those studies.

To conclude, the exemplars of the same relational concepts can vary in typicality and the second-order relational similarity between them accounts better for the systematic differences in the speed and the accuracy of the relational processing. This indicates a graded internal structure of the relational concepts and constrains the relational representation debate toward relational instead of semantic or role-similarity. Finally, the current dataset could be an important benchmark against which to test competing models of relational processing.

\section{Appendix: Instructions for the similarity ranking task}

Description: Full translated instructions for the relational, role and semantic similarity ranking task. Instructions were originally presented in Bulgarian.

Before each block, participants were instructed in detail about as to how to rank the exemplars in each trial. The same example was used for the instructions in order to highlight the difference between criteria:

BASE: GIANT DWARF

TARGETS: PEAK HILL, APARTMENTBUILDING HOUSE, SKYSCRAPER HUT 


\section{I. Ranking by relational similarity}

"Each of the exemplars in the next block contains word pairs that are related in similar ways - the two words in each pair are examples of a specific relation. In this example, the relation in each pair is " $X$ is higher than $Y$ " - the giant is higher than the dwarf, the peak is higher than the hill, etc. For each of the trials in the next block you have to rank the targets depending on how relationally similar they are to the base, i.e., to the degree that the words in each target hold a relation that is similar to the relation in the base pair."

"For example, in the word pairs PEAK HILL and SKYSCRAPER HUT the first word is always higher than the second, which is not necessarily true for the pair APARTMENTBUILDING HOUSE. The pair SKYSCRAPER HUT seems to be the most relationally similar to the base (i.e. GIANT DWARF) - a skyscraper is not only higher than a hut, but it can also be thought of as the opposite of a hut, just as a giant is in a way the opposite of a dwarf. For that reason, SKYSCRAPER HUT could be ranked as most relationally similar to the base, PEAK HILL can be ranked second, and APARTMENTBUILDING HOUSE - last. Rank the pairs according to what seems most logical to you. In each trial the base will define the relation. Think about that relation while you rank the target pairs."

\subsection{Ranking by role similarity of word I}

"Words can play different roles depending on the context in which they appear. Each of the trials in the next block contains words that play similar roles in each word pair. In each trial you have to rank the targets depending on how similar the role of first word in each target pair is to the role of the first word in the base, i.e., the word GIANT in the current example."

"In this example, a skyscraper, just like a giant, is the highest entity of its class (i.e., buildings and living beings). In contrast, a peak or an apartment building, while generally high, are not necessarily the highest in their class. Because of this, SKYSCRAPER should be ranked as having the most similar role to GIANT. We could rank PEAK in second place, because it is defined as "a point on earth's surface that is higher than the surrounding terrain". Finally, an APARTMENTBUILDING should be ranked last, because it is not necessarily the highest building. Rank according to what seems most logical to you. In each

trial the first word in the base will define the role. Think about that role while you rank the targets."

\subsection{Ranking by role similarity of word 2}

"Words can play different roles depending on the context in which they appear. Each of the trials in the next block contains words that play similar roles in each word pair. In each trial you have to rank the targets depending on how similar the role of second word in 
each target pair is to the role of the second word in the base, i.e., the word DWARF in the current example."

"In this example, a HUT, just like a DWARF, is the shortest entity of its class. For this reason, it should be ranked first, as having the most similar role to DWARF, because HUT is the best example among the targets for the role "shortest'. HILL is a small extension above the ground, which is a less similar to the role "shortest", so it should be ranked second. In last place we could rank HOUSE, because even though a house is generally not the highest building, it is also not the shortest one and thus it is not a good example of this role. Rank according to what seems most logical to you. In each trial the second word in the base will define the role. Think about that role while you rank the targets."

\subsection{Ranking by semantic similarity of word I}

"For each of the trials in the next block, rank the word pairs depending on how much the first word in each target pair is semantically similar to the first word in the base, i.e. with GIANT in this example."

"Inasmuch as giants are associated in various myths, legends and fairytales with beings that live in forests and mountains, the word PEAK seems to be the most semantically related word with GIANT in this example. In second place we could put SKYSCRAPER, because like GIANTS it is something tall and towering. The word APARTMENTBUILDING seems to be the least semantically similar to the GIANT. Rank according to what seems most logical to you. Think about the first word in the base and its meaning while you rank the targets."

\subsection{Ranking by semantic similarity of word 2}

"For each of the trials in the next block, rank the word pairs depending on how much the second word in each target pair is semantically similar to the second word in the base, i.e. with DWARF in this example."

"Inasmuch as dwarves are beings that live in forests, hills and underground, the word HILL seems to be the most semantically similar to DWARF in this example. In second place we could rank the word HUT, because it is a small building associated with poverty and being destitute, which people also with DWARVES as being small, insignificant and useless. The word HOUSE should rank third, because it is not semantically related at all to DWARF. Rank according to what seems most logical to you. Think about the first word in the base and its meaning while you rank the targets." 


\section{References}

Anderson, J. R. (1983). A spreading activation theory of memory. Journal of Verbal Learning and Verbal Behavior, 22(3), 261-295.

Asmuth, J., \& Gentner, D. (2016). Relational categories are more mutable than entity categories. The Quarterly Journal of Experimental Psychology, 1-19.

Bunge, S. A., Wendelken, C., Badre, D., \& Wagner, A. D. (2005). Analogical reasoning and prefrontal cortex: evidence for separable retrieval and integration mechanisms. Cerebral Cortex, 15(3), 239-249.

Chaffin, R. (1992). The concept of a semantic relation. Frames, Fields and Contrasts, 253288.

Chaffin, R., \& Herrmann, D. J. (1984). The similarity and diversity of semantic relations. Memory \& Cognition, 12(2), 134-141.

Chaffin, R., \& Herrmann, D. J. (1989). Retrieval and comparison processes in part-whole decisions. The Journal of General Psychology, 116(4), 393-406.

Chiang, J. N., Peng, Y., Lu, H., Holyoak, K. J., \& Monti, M. M. (2019). Distributed Code for Semantic Relations Predicts Neural Similarity. BioRxiv, 596726.

Collins, A. M., \& Loftus, E. F. (1975). A spreading-activation theory of semantic processing. Psychological Review, 82(6), 407.

Collins, A. M., \& Quillian, M. R. (1969). Retrieval time from semantic memory. Journal of Verbal Learning and Verbal Behavior, 8(2), 240-247.

Doumas, L. A., \& Hummel, J. E. (2005). Approaches to modeling human mental representations: What works, what doesn't and why. The Cambridge Handbook of Thinking and Reasoning, Ed. KJ Holyoak \& RG Morrison, 73-94.

Doumas, L. A., Hummel, J. E., \& Sandhofer, C. M. (2008). A theory of the discovery and predication of relational concepts. Psychological Review, 115(1), 1.

Dunbar, K. (2001). The analogical paradox: Why analogy is so easy in naturalistic settings yet so difficult in the psychological laboratory. The Analogical Mind: Perspectives from Cognitive Science, 313-334.

Epstein, M. L., Phillips, W. D., \& Johnson, S. J. (1975). Recall of related and unrelated word pairs as a function of processing level. Journal of Experimental Psychology: Human Learning and Memory, 1(2), 149.

Estes, Z., \& Jones, L. L. (2006). Priming via relational similarity: A copper horse is faster when seen through a glass eye. Journal of Memory and Language, 55(1), 89-101.

Forbus, K. D., Gentner, D., \& Law, K. (1995). MAC/FAC: A Model of Similarity-Based Retrieval. Cognitive Science, 19(2), 141-205.

Gagné, C. L., Spalding, T. L., \& Ji, H. (2005). Re-examining evidence for the use of independent relational representations during conceptual combination. Journal of Memory and Language, 53(3), 445-455.

Gentner, D. (1983). Structure-mapping: A theoretical framework for analogy. Cognitive Science, 7(2), 155-170. 
Gentner, D. (2010). Bootstrapping the mind: Analogical processes and symbol systems. Cognitive Science, 34(5), 752-775.

Gentner, D., \& Kurtz, K. J. (2005). Relational categories. Categorization inside and Outside the Lab, 151-175.

Gentner, D., \& Markman, A. B. (1997). Structure mapping in analogy and similarity. American Psychologist, 52(1), 45.

Gick, M. L., \& Holyoak, K. J. (1980). Analogical problem solving. Cognitive Psychology, 12(3), 306-355.

Goldstone, R. L., Medin, D. L., \& Gentner, D. (1991). Relational similarity and the nonindependence of features in similarity judgments. Cognitive Psychology, 23(2), 222-262.

Halford, G. S., Wilson, W. H., \& Phillips, S. (1998). Processing capacity defined by relational complexity: Implications for comparative, developmental, and cognitive psychology. Behavioral and Brain Sciences, 21(6), 803-831.

Halford, G. S., Wilson, W. H., \& Phillips, S. (2010). Relational knowledge: the foundation of higher cognition. Trends in Cognitive Sciences, 14(11), 497-505.

Hofstadter, D., \& Sander, E. (2013). The talent so fundamental that it is fuel to our minds. New Scientist, 218(2915), 30-33.

Holyoak, K. J. (2007). Relations in semantic memory: Still puzzling after all these years. Memory and Mind: A Festschrift for Gordon H. Bower. Erlbaum, 141-158.

Holyoak, K. J. (2012). Analogy and relational reasoning. The Oxford Handbook of Thinking and Reasoning, 234-259.

Hummel, J. E., \& Holyoak, K. J. (1997). Distributed representations of structure: A theory of analogical access and mapping. Psychological Review, 104(3), 427.

Ichien, N., Lu, H., \& Holyoak, K. J. (2019). Individual differences in judging similarity between semantic relations. In A. Goel, C. Seifert, \& C. Freksa (Eds.), Proceedings of the 41st Annual Meeting of the Cognitive Science Society. Austin, TX: Cognitive Science Society.

Jamrozik, A., \& Gentner, D. (2020). Relational labeling unlocks inert knowledge. Cognition, $196,104146$.

Jones, M., \& Love, B. C. (2007). Beyond common features: The role of roles in determining similarity. Cognitive Psychology, 55(3), 196-231.

Jurgens, D. A., Turney, P. D., Mohammad, S. M., \& Holyoak, K. J. (2012). Semeval-2012 task 2: Measuring degrees of relational similarity. In Proceedings of the First Joint Conference on Lexical and Computational Semantics-Volume 1: Proceedings of the main conference and the shared task, and Volume 2: Proceedings of the Sixth International Workshop on Semantic Evaluation (pp. 356-364). Association for Computational Linguistics.

Keane, M. (1987). On retrieving analogues when solving problems. The Quarterly Journal of Experimental Psychology Section A, 39(1), 29-41. 
Kokinov, B., \& Petrov, A. (2001). Integrating memory and reasoning in analogy-making: The AMBR model. The Analogical Mind: Perspectives from Cognitive Science, 59-124.

Leech, R., Mareschal, D., \& Cooper, R. P. (2008). Analogy as relational priming: A developmental and computational perspective on the origins of a complex cognitive skill. Behavioral and Brain Sciences, 31(04).

Lu, H., Chen, D., \& Holyoak, K. J. (2012). Bayesian analogy with relational transformations. Psychological Review, 119(3), 617-648.

Lu, H., Liu, Q., Ichien, N., Yuille, A. L., \& Holyoak, K. J. (2019a). Seeing the meaning: Vision meets semantics in solving pictorial analogy problems. Proceedings of the Annual Conference of the Cognitive Science Society.

Lu, H., Wu, Y. N., \& Holyoak, K. J. (2019b). Emergence of analogy from relation learning. Proceedings of the National Academy of Sciences, 116(10), 4176-4181.

Mahon, B. Z., \& Hickok, G. (2016). Arguments about the nature of concepts: Symbols, embodiment, and beyond. Psychonomic Bulletin \& Review, 23(4), 941-958.

Medin, D. L., Goldstone, R. L., \& Gentner, D. (1993). Respects for similarity. Psychological Review, 100(2), 254-278.

Mervis, C. B., Catlin, J., \& Rosch, E. (1976). Relationships among goodness-of-example, category norms, and word frequency. Bulletin of the Psychonomic Society, 7(3), 283284 .

Murphy, G. L. (2002). The big book of concepts. Cambridge, Mass. [u.a.: MIT Press.

Nelson, D. L., McEvoy, C. L., \& Schreiber, T. A. (2004). The University of South Florida free association, rhyme, and word fragment norms. Behavior Research Methods, Instruments, \& Computers, 36(3), 402-407.

Penn, D. C., Holyoak, K. J., \& Povinelli, D. J. (2008). Darwin's mistake: Explaining the discontinuity between human and nonhuman minds. Behavioral and Brain Sciences, 31(2), 109-130.

Popov, V., \& Hristova, P. (2015). Unintentional and efficient relational priming. Memory \& Cognition, 43(6), 866-878.

Popov, V., Hristova, P., \& Anders, R. (2017). The Relational Luring Effect: Retrieval of Relational Information During Associative Recognition. Journal of Experimental Psychology. General.

Popov, V., Zhang, Q., Koch, G. E., Calloway, R. C., \& Coutanche, M. N. (2019). Semantic knowledge influences whether novel episodic associations are represented symmetrically or asymmetrically. Memory \& Cognition, 1-15.

Raynal, L., Clément, E., \& Sander, E. (2020). Are Superficially Dissimilar Analogs better retrieved than Superficially Similar Disanalogs? Acta Psychologica, 203, 102989.

Rosch, E. (1975). Cognitive representations of semantic categories. Journal of Experimental Psychology: General, 104(3), 192.

Rosch, E., \& Mervis, C. B. (1975). Family resemblances: Studies in the internal structure of categories. Cognitive Psychology, 7(4), 573-605. 
Rosch, E., Simpson, C., \& Miller, R. S. (1976). Structural bases of typicality effects. Journal of Experimental Psychology: Human Perception and Performance, 2(4), 491.

Ross, B. H. (1989). Distinguishing types of superficial similarities: Different effects on the access and use of earlier problems. Journal of Experimental Psychology: Learning, Memory, and Cognition, 15(3), 456-468.

Silberman, Y., Bentin, S., \& Miikkulainen, R. (2007). Semantic Boost on Episodic Associations: An Empirically-Based Computational Model. Cognitive Science, 31(4), 645-671.

Sloman, S. A., \& Rips, L. J. (1998). Similarity as an explanatory construct. Cognition, 65(2), 87-101.

Smith, E. E., Shoben, E. J., \& Rips, L. J. (1974). Structure and process in semantic memory: A featural model for semantic decisions. Psychological Review, 81(3), 214.

Spellman, B. A., Holyoak, K. J., \& Morrison, R. G. (2001). Analogical priming via semantic relations. Memory \& Cognition, 29(3), 383-393.

Tversky, A. (1977). Features of similarity. Psychological Review, 84(4), 327. 